\title{
Cambio en las estructuras de significación en organizaciones de pequeños productores. Una comprensión desde la teoría institucional ${ }^{1}$
}

\author{
Luri Suárez Pineda ${ }^{2}$ \\ Universidad Santo Tomás. \\ luri.suarez@usantoto.edu.co \\ https://orcid.org/0000-0002-9770-4965 \\ Marlén Suárez Pineda ${ }^{3}$ \\ Universidad Pedagógica y Tecnológica de Colombia. \\ marlen.suarez@uptc.edu.co \\ https://orcid.org/0000-0003-2104-3977
}

\begin{abstract}
How to cite this paper:
Suárez-Pineda, Luri; Suárez-Pineda, Marlén (2020). Cambio en las estructuras de significación en organizaciones de pequeños productores. Una comprensión desde la teoría institucional. Revista Encuentros, Universidad Autónoma del Caribe. Vol. 18-01.
\end{abstract}

Doi: 10.15665/encuent.v18i01.2162

Recibido: 23 de octubre de 2019 / Aceptado: 14 de diciembre de2019

\section{RESUMEN}

La perspectiva teórica institucional que promete superar la estrechez comprensiva del racionalismo instrumental orientó la aproximación al fenómeno del cambio en organizaciones de pequeños productores del eslabón primario de la cadena láctea en Colombia, con ocasión de un instrumento de política pública que pretendía aliviar los impactos de la apertura comercial del sector. Este artículo se ocupa del cambio en las estructuras de significación, comprendidas como constructo social, en los pequeños productores del departamento de Boyacá. Se llevó a cabo un estudio cualitativo de carácter interpretativo cuyo hallazgo se concreta en que los arreglos institucionales en los que se fundamentó el mecanismo de apoyo estatal, no lograron la construcción de sentido entre los sujetos beneficiarios, y en consecuencia, no ocurrieron los cambios esperados.

Palabras clave: Cambio organizacional, teoría institucional, política pública.

\section{Change in the structures of significance in organizations of small producers. An understanding from institutional theory}

\begin{abstract}
The institutional theoretical perspective that promises to overcome the comprehensive narrowness of instrumental rationalism, guided the approach to the phenomenon of change in organizations of small producers of the primary link of the dairy chain in Colombia, on the occasion of a public policy instrument that sought to alleviate the impacts of the commercial opening of the sector. This article deals with the change in the structures of significance, understood as social construct, in small producers in the department of Boyacá. A qualitative interpretive study was carried out whose finding is specified in that the institutional arrangements on which the state support mechanism was based, did not achieve the construction of meaning among the beneficiary subjects, and consequently, the expected changes did not occur .
\end{abstract}

Keywords: Organizational change, institutional theory, public policy.

1 Resultado parcial del proyecto de investigación titulado "El cambio organizacional en el sector lácteo del departamento de Boyacá. Un análisis desde la política pública"

2 Magíster en Administración.

3 Magíster en Administración. Estudiante de doctorado en Administración de la Universidad Autónoma de Querétaro. 


\title{
Mudança nas estruturas de significação em organizações de pe- quenos produtores. Uma compreensåo desde a teoria innstitucional
}

\begin{abstract}
RESUMO
A perspectiva teórica institucional que promete superar a carência compreensiva do racionalismo instrumental, orientou a aproximação ao fenômeno da mudança em organizações de pequenos produtores do elo primário da cadeia láctea na Colômbia, com ocasião de um instrumento de política pública que pretendia aliviar os impactos da abertura comercial do setor. Este artigo ocupase da mudança nas estruturas de significação, compreendidas como constructo social, nos pequenos produtores do departamento de Boyacá. Levou-se a cabo um estudo qualitativo de carácter interpretativo cuja descoberta concreta-se em que os consertos institucionais nos quais fundamentou-se o mecanismo de apoio estatal, não lograram a construção de sentido entre os sujeitos beneficiários, e em consequência, não ocorreram as mudanças esperadas.
\end{abstract}

Palavras chave: Mudança organizacional, teoria institucional, política pública.

\section{Introducción}

A partir de la última década del siglo 20 ha desfilado un complejo entramado de decisiones gubernamentales que buscan la conformidad con el pensamiento dominante a favor del libre mercado. Estas lógicas señalan la conveniencia de los tratados comerciales internacionales que suponen el desmonte de mecanismos de política comercial de carácter proteccionista y proponen a los sectores productivos jugar en escenario de libre competencia, exhortados por los postulados de la competitividad basada en la eficiencia y la productividad. Sectores productivos tradicionales en el país como el lácteo exhiben la necesidad de la intervención del Estado para superar "altos costos de producción, baja productividad en relación con los principales actores internacionales, dispersión en la producción primaria, mínimo nivel de asociatividad en los eslabones de la cadena, alta informalidad en la comercialización y transformación" (Conpes 3675 de 2010, p. 4), entre otros retos planteados para responder a los cambios en el contexto.

El gobierno nacional consciente de las asimetrías entre las capacidades competitivas domésticas y las de los nuevos actores en el mercado, promulgó instrumentos de política para acompañar la superación de las debilidades; el Consejo Nacional de Política Económica y Social-CONPES- aprueba el documento conpes 3675 de 2010 y con él se propone un plan de acción para el sector lácteo financiado con recursos del presupuesto general de la nación y de cooperación con la Unión Europea.

Desde el nivel central se definieron las acciones a desarrollar en los territorios; el departamento de Boyacá fue incorporado al plan con distintas estrategias, entre otras, la promoción de la organización de los pequeños productores en esquemas asociativos que posibilitaran el mejoramiento competitivo. Para estas organizaciones el programa conpes dispuso de un plan de capacitación y un kit de maquinaria, equipos e insumos, en el orden de rotocultivador, desgrosadora, tanque de refrigeración para acopio, baldes, plantas, semillas, entre otros.

Esta investigación se ocupa de observar el fenómeno del cambio con ocasión del conpes 3675 de 2010, en las organizaciones de pequeños productores en Boyacá, particularmente en la región central, con una lente antipositivista que posibilita comprensiones desde los individuos que experimentan la acción y no desde el observador, asumiendo el carácter voluntarista del ser humano (Burrel y Morgan, 1979). Los cambios de tipo racional reflejados en las cifras de la competitividad se dejan para otros intereses investigativos.

Las convergencias simbólicas entendidas de acuerdo con Bormann(1983) como los significados compartidos que emergen de las fantasías que se recrean habitualmente entre los miembros de un grupo y que les permiten experimentar las mismas emociones, es el concepto utilizado en este trabajo para señalar las 
coincidencias interpretativas que se hallan entre los individuos escuchados.

Se encuentra una evidencia empírica de la tesis de March (1994) en relación con la concepción de orden en la toma de decisiones, en la realidad el tomador de decisiones en la organización obra en un plano de incertidumbre, ambigüedad y confusiones antes que, de causalidad, objetividad y clara intencionalidad, razón por la que muchos planes y políticas sistemáticamente racionales son difíciles de cumplir. Esta postura confluye con el concepto de racionalidad limitada de Simon (1988).

\section{La teoría institucional y el cambio organizacional como constructo social}

Desde la perspectiva racionalista se pueden citar varias corrientes de la teoría organizacional que sugieren posturas en torno al cambio en la organización con un carácter determinista, considerándosele como un proceso prescrito y controlado. Para Weber (2002) "la precisión, la rapidez, la univocidad, la oficialidad, la continuidad, la discreción, la uniformidad, la rigurosa subordinación, el ahorro de fricciones y de costas objetivas y personales, son infinitamente mayores en una administración severamente democrática” (p. 731). Ibarra y Montaño (1987) refiriéndose a la obra de Weber consideran que la racionalidad implica el diseño y la construcción de un sistema administrativo mediante la división del trabajo y la coordinación de actividades bajo la inspiración de la mayor productividad.

Las corrientes tradicionales asumen la noción del cambio organizacional desde un paradigma lineal funcionalista, en donde los procesos de cambio son deliberadamente diseñados y controlados en dirección a los propósitos de la organización, y en consecuencia, el manejo de la resistencia al cambio se convierte en un reto para la administración, prevalece un afán de modificar la realidad de acuerdo con los propósitos económicos de la organización, desconociendo la naturaleza propia de los sujetos y la complejidad de la realidad (Muñoz, 2010).

Una mirada alternativa emerge de la postmodernidad, en donde los límites del contexto son difusos (tecnología, alianzas, tercerización), la estructura hace símil con anarquías organizadas, predomina lo coyuntural y emergente, el control se basa en el autodominio y la ambigüedad, y la singularidad sustituyen la especificación y la universalidad. Autores en esta perspectiva, que encuadra más en el interpretativismo, se cuentan los defensores de los estudios organizacionales y los críticos de la teoría organizacional convencional, pueden mencionarse en esta línea los trabajos de Simon (1988) y March (1994), que parten del principio que el hombre toma decisiones en el marco de una racionalidad limitada y bajo circunstancias de incertidumbre y ambigüedad. La realidad organizacional se aprecia como un espacio multidimensional que supera la simplicidad de lo ordenado y funcional, para considerar la intervención de diversos actores con intereses propios (De la Rosa, 2002), por lo tanto las organizaciones no pueden pensarse por partes, sino desde la complejidad que implica la interacción de muchos elementos como lo señala la figura 1 , sin regularidades ni causalidades.

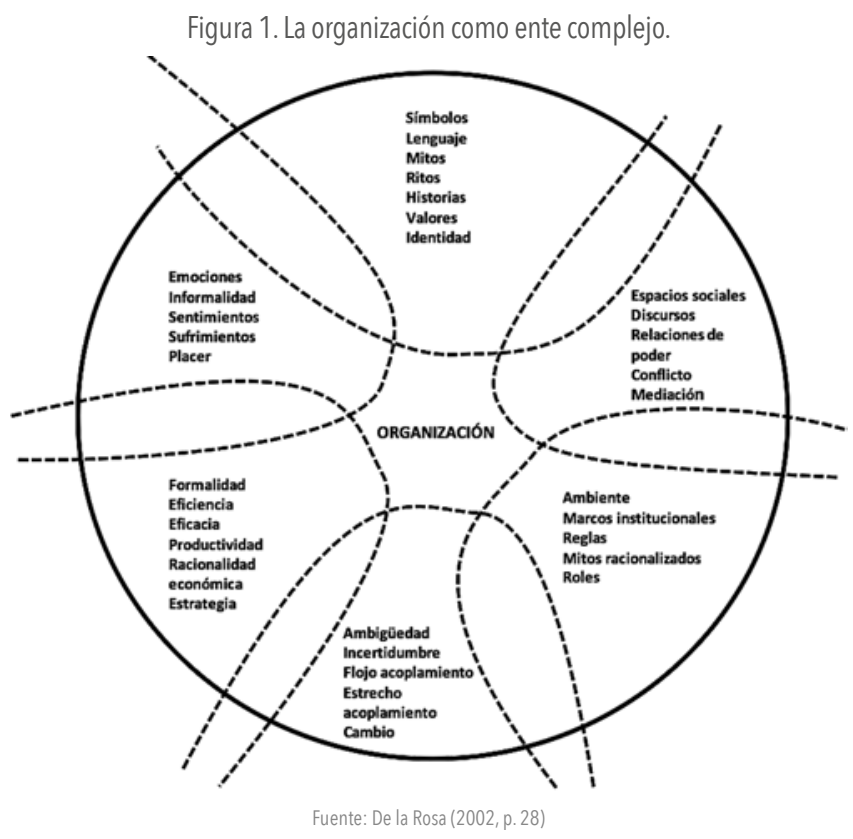

En la búsqueda de marcos conceptuales más amplios que permitan explicar la realidad observable en las organizaciones, que estén más ligados a la conciencia humana y que permitan entender el cambio organizacional desde la lente del cambio social, se intenta desde la teoría del institucionalismo, allí donde emergen las nociones de cultura y de poder, mismas que se ubican en la sociología de la organización (Crozier y Friedberg, 1990) para comprender el cambio organizacional como un proceso de construcción colectiva por medio del cual los miembros de una determinada colectividad inventan nuevas formas de interactuar. 
La teoría institucional se convierte en un rompimiento epistemológico, en una nueva manera de percibir el mundo organizacional, de pensar y de estudiar los problemas con un poder explicativo para interpretar problemas específicos de comportamiento de los actores organizacionales (Alvarado, 2006). Es un enfoque teórico iniciado en el trabajo de Selznick (1949) titulado la Autoridad del Valle de Tennessee, en donde se pone en evidencia que patrones sociales coexisten junto con los elementos técnicos y económicos, para definir la cotidianidad de las organizaciones.

Para Jepperson (1999), la institución y la institucionalización son conceptos centrales en la sociología general. "La institución representa un orden o patrón social que ha alcanzado cierto estado o propiedad" (p.195); cuando las desviaciones del patrón son contrarrestadas sistemáticamente por medio de controles socialmente construidos que se autoactivan, se afirma que el patrón está institucionalizado. De este modo institucionalismo se puede definir como argumentos que se caracterizan por restricciones de orden superior impuestas por realidades construidas socialmente. El concepto de reglas institucionalizadas según Berger y Luckman (1995), refiere a tipificaciones o interpretaciones intercambiadas, tales reglas se apoyan en la opinión pública o en la fuerza de la ley. "La institución, con su conjunto de acciones programadas, se asemeja al libreto no escrito de una obra teatral. La realización de la obra depende de que actores de carne y hueso desempeñen reiteradamente los roles prescritos" (Berger y Luckmann, 1995, p. 99).

El nuevo institucionalismo en la teoría organizacional representa el rechazo de los modelos racionales y concibe que las convenciones entre los seres humanos toman estatus con carácter de norma en el pensamiento y la acción social. Para Dimaggio y Powell (1999) en la ciencia política se dio origen a los nuevos enfoques institucionalistas, en la economía el nuevo institucionalismo nació como reacción a los excesos originados en la economía neoclásica que privilegia la elección racional de los actores, ignorando costos de transacción y restricciones y defendiendo el postulado de que el equilibrio es derivado de la selección de acuerdo con la racionalidad maximizadora.
Los neoinstitucionalistas sociológicos se apartan de las teorías de la elección racional y admiten que la racionalidad de los actores está limitada por las capacidades cognitivas del ser humano. En esta perspectiva se reconoce la existencia de un contexto institucional, conformado por "mitos, creencias y rutinas, que se convierten en generadoras potenciales de pautas de comportamiento socialmente aceptadas que propician en las organizaciones una tendencia a la uniformidad y a la estabilidad, más que a la diversidad y al cambio" (Alvarado, 2006, p. 15). Si bien los neoinstitucionalistas de la corriente económica y los de la corriente sociológica consideran a las instituciones como creación humana, los primeros la asumen como el resultado de acciones intencionales que realizan individuos racionales orientados instrumentalmente, mientras para los segundos las instituciones son patrones sociales que tienen un proceso de reproducción.

Dimaggio y Powell (1999) desarrollan los conceptos de campo organizacional, legitimidad e isomorfismo organizacional, refiriendo el primero al conjunto de organizaciones que pertenecen a una industria y que "constituyen un área reconocida de la vida institucional” (p. 106), integrado por productores, proveedores, competidores, consumidores y reguladores. La legitimidad entonces se deriva del grado de institucionalización, una organización es más legítima cuanto más institucionalizada se encuentre, es decir cuanto más refleje alineación cultural, apoyo normativo y consonancia con las reglas y leyes del campo (Cruz, Prado y Díez, 2014).

El isomorfismo hace relación al proceso de homogenización, según el cual las organizaciones se van mostrando cada vez más similares en su misión, objetivos, estructura formal y cultura, como consecuencia de los arreglos institucionales que ejercen presión sobre el campo. Los mismos autores identifican tres mecanismos de cambio institucional isomorfo: coercitivo, mimético y normativo; corresponde a tres fuerzas que conducen a las organizaciones a mostrar cada vez mayor conformidad con arreglos institucionales y en consecuencia más similitud con las demás dentro del campo. En el isomorfismo coercitivo la presión que homogeniza se deriva de organismos reguladores o generadores de prescripciones condicionantes, como el gobierno o un comprador con alto poder de negociación; 
el isomorfismo mimético es derivado de la propensión a imitar las organizaciones más legitimadas en el campo, como mecanismo para superar la incertidumbre, y en el isomorfismo normativo la presión proviene de los patrones institucionalizados a nivel de la profesión u oficio.

Las estructuras formales institucionalizadas, definidas como organizaciones formales son: "sistemas de actividades controladas y coordinadas que surgen cuando el trabajo es incorporado en redes complejas de relaciones técnicas e intercambios que traspasan fronteras" (Meyer y Rowan, 1999, p. 79). Las evidencias empíricas contradicen las teorías racionales prevalecientes de la estructura formal, precisando que existe diferencia entre la estructura organizacional y las actividades de trabajo diario. No cabe duda que los mercados económicos favorecen la racionalidad y que el trabajo coordinado formalmente tiene ventajas competitivas, además, las teorías dominantes suponen que el éxito de las organizaciones descansa en la coordinación y el control de las actividades; por otra parte, investigaciones empíricas han puesto en evidencia que las organizaciones no mantienen una cotidianidad ajustada a sus planes y no siguen prescripciones de la estructura formal (Meyer y Rowan, 1999). La propuesta de la teoría institucional es reconocer los elementos socioculturales construidos históricamente presentes en la acción humana, sin excluir por supuesto el cálculo racional maximizador (Tolbert y Zucker, 1996).

El estudio del cambio organizacional en organizaciones de productores de leche, debe abordarse desde un enfoque ecléctico, en donde conversen las teorías de corte determinista-racionalista con las de corte interpretativo, es decir la teoría organizacional convencional con los estudios organizacionales, para encontrar mayor riqueza en las explicaciones sobre el origen, el proceso y la velocidad de los cambios en este tipo de organizaciones.

\section{Metodología}

Esta es una investigación de enfoque cualitativo que se apoya en herramientas de tipo interpretativosubjetivo, siguiendo las definiciones de los estudios fenomenológicos de Creswell (2007).
Las organizaciones consideradas fueron las ubicadas en la zona centro del departamento de Boyacá, atendiendo la manifestación de interés en esta investigación por parte de la Federación de Ganaderos de Boyacá, organismo que representa el gremio en el departamento. A partir de la base de datos de la Secretaría de Fomento Agropecuario de la Gobernación de Boyacá, que contenía el listado de organizaciones de productores de leche beneficiados con los recursos del Consejo Nacional de Política Económica y Social -CONPES- a través del Conpes Lechero No. 3675, se seleccionaron las organizaciones de productores que hacían parte de un programa de salud animal preventiva de la Federación citada. Luego de consultar a los representantes legales y recibir la confirmación de aceptar participar en la investigación, se determinó un total de 21 organizaciones ubicadas en 15 municipios de la región central del departamento, como grupo participante en la investigación.

Las unidades de observación fueron los representantes legales de cada organización, miembros de juntas directivas o consejos de administración y asociados. La aproximación tuvo un carácter emic en la medida que las comprensiones emergieron del punto de vista de los agentes involucrados en el fenómeno social organizacional, para lo cual fue necesario aplicar entrevistas semiestructuradas, grupos focales y observaciones no participantes, estas últimas para reconocer los discursos y rituales expuestos en la cotidianidad de los sujetos, como opción para comprender sus comportamientos frente a las propuestas de cambio presentadas por el gobierno.

La recolección de la información y el tratamiento de la misma, estuvo orientada por la búsqueda de respuestas a la pregunta de investigación: ¿de qué manera tuvo lugar el cambio organizacional en asociaciones de pequeños productores de leche, por razón de haber sido beneficiarios del programa conpes 3675 de 2010?; para el efecto se definieron previamente dimensiones de análisis que emergieron de los preceptos teóricos y de la mirada ecléctica del investigador para observar tanto los elementos de tipo racional instrumental como las construcciones sociológicas. Este artículo está referido a los hallazgos en torno a una de las la dimensiones: cambios en las estructuras de significación de los sujetos que conforman las organizaciones. 


\section{Resultados}

El análisis de la información recaudada para identificar los cambios en las estructuras de significación de los sujetos sociales organizacionales, permitió identificar convergencias simbólicas (Bormann, 1983), que permitieron emitir la siguiente proposición:

Las organizaciones de pequeños productores de leche no son organizaciones institucionalizadas, aunque la estructura de significados compartida actúa como elemento homogenizador.

Los arreglos hegemónicos dictados por el mercado y apropiados por el Estado para instrumentar la política pública, en el orden de la competitividad, la eficiencia y la productividad, no han logrado convertirse en patrones condicionantes de la gestión de las organizaciones de pequeños productores de leche, por ello se las puede considerar como organizaciones no institucionalizadas; las racionalidades que subyacen son propias de las estructuras de significación compartidas y construidas a lo largo de la historia de las comunidades. La evidencia empírica es que lo previsto por Dimaggio y Powell (1999) sobre las presiones isomórficas de tipo coercitivo, mimético y normativo, no son en este caso, las responsables de que las estructuras y prácticas en las organizaciones, se guarden alto grado de similitud.

La investigación reportó que las formas de relacionarse, interactuar, planear, tomar decisiones, al interior de las organizaciones, es similar para todos los casos observados, pero no porque el mercado, los entes reguladores, los casos exitosos o arreglos propios del oficio, actúen para ese isomorfismo. Los elementos que confluyen para que estas organizaciones se gestionen de manera similar, son los esquemas interpretativos que comparten acerca del largo plazo, de sí mismos como productores, del papel Estado, de la organización y el asociado, del mercado, para señalar los más relevantes observados.

Las convergencias simbólicas reflejadas en las entrevistas y grupos focales en torno a estos aspectos, se presentan de la siguiente manera:

\section{a). Alto interés por los resultados mediáticos desestimando el largo plazo}

Los relatos ponen en evidencia que el largo plazo no es un asunto que ocupe las agendas de las organizaciones ni de los productores individualmente, la preocupación se centra en cómo resolver los requerimientos inmediatos que solo son valorados en términos de recursos financieros o físicos, con menosprecio por el conocimiento y las habilidades que posibilitan consensos hacia el futuro.

Un asociado refiriéndose a las capacitaciones recibidas por el conpes relata: "mucho protocolo que no estamos dispuestos a hacer... no estamos dispuestos a hacer por tan poquitas vacas que tenemos, empezando por las distancias para llevar una o dos vacas hasta el establo". Se muestra común en la experiencia de las organizaciones que los asociados reflejaron alta motivación ante la expectativa de recursos provenientes del Estado, motivación que no fue sostenible, no alcanzó para apropiar las capacitaciones sobre nuevas prácticas y hacer apuestas comunes por el largo plazo. Un directivo comenta:

"Nosotros pensamos ya cancelarnos, y volver a trabajar cada cual independiente, no salieron con nada. Yo duré en una capacitación en Tunja, nos tuvieron 15 días...allá hay ingenieros y le van a enseñar a uno que es criado en el campo y sabe cómo es para tener una buena vaca”, uno ya sabe vacunar e inseminar, entonces una capacitación para qué...No hay recursos del gobierno, solo que vaya para Tunja a recibir capacitaciones...cuando echaron a no dar nada la gente se desmotivó".

\section{b). Baja autoestima en su condición de pequeños productores}

Esta autovaloración impide visualizarse como empresarios y se antepone a las lógicas del mercado que señalan entre otras, que a través de la organización podrían hacer economías de escala y elevar su poder de negociación frente al cliente. En un grupo focal con miembros de una de las asociaciones, alguien comenta: "Las herramientas que nos facilitó el conpes son buenas para ensilar y cosechar, pero nosotros somos pequeños ganaderos, ni ensilamos ni cosechamos, lástima de esos recursos", otro participante haciendo una autocrítica expresa:

"Como somos pequeños estamos convencidos que nuestras tierras no sirven, pero los que compran viniendo de afuera sí las hacen producir. Nuestros hijos se van y nosotros vendemos la tierra a forasteros que sí traen estrategias exitosas, hasta italianos, franceses y canadienses". 


\section{c). Representación compartida en torno al papel del Estado}

Directivos y asociados convergen en la idea de que al Estado le corresponde un rol asistencialista con el deber de dar recursos a los pequeños productores para hacer viable su actividad económica, contrariando los patrones del libre mercado. En un grupo focal con asociados de una de las organizaciones se escucha: "el Estado no nos garantiza precios, no hay acompañamiento en el mercado", esta afirmación es complementada por distintas voces que señalan la escasa pertinencia de los recursos físicos entregados y de las capacitaciones impartidas; uno de los participantes expone:

La maquinaria que nos trajo el conpes la tenemos arrumada en nuestras organizaciones, no le estamos dando uso, nuestras actividades no la requieren. Los ganaderos especializados dicen que el Estado le dio pan al que no tiene dientes, más bien pensamos en alquilársela a ellos.

En entrevista, un gerente refiere "Los asociados se muestran desmotivados esperando que el Estado les traiga recursos".

La imagen de Estado protector concurre con la percepción de que hay afán de ejecutar los recursos públicos sin importar la eficacia de estas acciones gubernamentales; en las entrevistas con directivos $\mathrm{y}$ asociados fueron recurrentes las expresiones de descontento, siendo las siguientes de mayor convergencia: problemas de los campesinos imaginados desde un escritorio, capacitadores incompetentes, equipamientos no correspondientes con las necesidades, interés centrado en la construcción de la evidencia, gobiernos locales ausentes y despreocupados por sumar a los propósitos de estos programas.

\section{d). La organización -asociación- no es considerada como estrategia para el propio desarrollo}

Persiste la idea de organización como ente que les representa y posibilita la recepción de ayudas externas, pero no es reconocida como estrategia para alcanzar cambios en sus actividades productivas y realidades socioeconómicas. La ausencia de compromiso de los asociados es la afirmación de mayor recurrencia en el curso del trabajo de campo. No se cumple con aportes financieros para el sostenimiento, no hay participación en las discusiones ni en la toma de decisiones, no se acatan compromisos de cambio en las prácticas del oficio; señalamientos de esta naturaleza son emitidos por al menos el $80 \%$ de los gerentes entrevistados. Un gerente afirma: "nos falta compromiso social, nos falta empoderarnos. Con lo del conpes faltó más contacto con la sociedad, hubo capacitación con afán". Refiriéndose a sus asociados un gerente comenta: "no acopian porque les exigen calidad y porque no quieren dar un peso para la asociación, es como el temor a la exigencia". Ante la pregunta sobre la toma de decisiones en la organización, un gerente que al mismo tiempo es asociado responde: "Yo he sido el representante legal desde que empezó la asociación, hace nueve años, porque no ha habido nadie más que se bandee con esto. Yo ya no quiero seguir más con esto, yo propongo que se cambie el líder".

Sobre el mismo particular un asociado relata: "A muchos no les sirve la maquinaria que les entregó el compes, esa maquinaria está guardada; nos llamamos asociación de lecheros pero no hacemos nada como asociados, nuestras acciones no tienen nada de asociación".

Salvo dos excepciones de las 21 organizaciones observadas, el gerente no cuenta con el respaldo de una junta directiva o consejo de administración, porque este organismo directivo no ejerce su rol; la participación de los asociados en las discusiones sobre el futuro de la organización es muy débil, no hay acuerdos para incorporar cambios en las formas de ejecutar las tareas. Los asociados dejan la responsabilidad de la gestión en cabeza del gerente, desconocen sus propios estatutos y en consecuencia desconocen la razón de ser de la organización así como los deberes y derechos como asociados, situación que se evidencia en las respuestas ante las preguntas en torno a los planes y proyectos, en el orden de "el que sabe es el gerente". El trabajo de campo aporta elementos para inferir que este comportamiento de los asociados está explicado por el nivel educativo que en promedio es educación primaria. En el $80 \%$ de las organizaciones observadas el gerente es un profesional, que viene respondiendo por la viabilidad legal de la asociación, atendiendo los compromisos con los organismos del gobierno, la mayoría de ellos expresó en la entrevista el deseo de no continuar al frente de esta labor, situación que incrementa la incertidumbre hacia el futuro porque en todos los casos no hay asociados interesados en asumir este rol como 
tampoco existen las capacidades intelectuales para ello. En tres organizaciones se encontró que el gerente es un profesional externo, son los únicos casos en los que existe un contrato laboral para este cargo y en consecuencia una remuneración salarial, tales casos corresponden a las organizaciones que acopian un volumen importante y comercializan con la industria formal.

Las comprensiones desde el gremio ganadero regional señalan la tenencia de la tierra como uno de los factores que sostienen este panorama, la propiedad es heredada a los hijos del productor agudizándose el microfundio, estructura que imposibilita economías de escala y especialización. El productor se ocupa de otros múltiples oficios a pequeña escala, como agricultura, porcicultura, avicultura, etc., para generar los ingresos mínimos que requiere su unidad familiar.
Se pudo evidenciar en la interacción con gerentes y asociados, que aquellas organizaciones que se originaron antes de la promulgación del conpes citado, como consecuencia de decisiones más autónomas del grupo, han podido construir algunas señales de capital social relativo a trabajo en equipo y participación en la recreación del futuro organizacional.

La Tabla 1 muestra la caracterización general de las organizaciones participantes en el estudio.

\section{e). Los patrones del mercado se interpretan como obligaciones ajenas}

Las representaciones convergen en que los actores industriales formales no les apoyan, hacen altas exigencias de calidad y no pagan por ella. Con escasas excepciones los asociados no reconocen la importancia de cumplir estándares de calidad en la leche, estos son

Tabla 1. Caracterización de las organizaciones participantes

\begin{tabular}{|c|c|c|c|c|c|c|c|c|}
\hline \multirow{3}{*}{ MUNICIPIO } & \multirow{3}{*}{ ORGANIZACIÓN } & \multicolumn{3}{|c|}{ CONSTITUCIÓN } & \multirow{2}{*}{\multicolumn{2}{|c|}{$\begin{array}{l}\text { ESCOLARIDAD PROMEDIO } \\
\text { DE LOS ASOCIADOS }\end{array}$}} & \multirow{2}{*}{\multicolumn{2}{|c|}{$\begin{array}{l}\text { CONDICIÓN DEL GERENTE } \\
\text { Y REPRESENT ANTE LEGAL }\end{array}$}} \\
\hline & & \multicolumn{2}{|c|}{ Conpes 3675 de 2010} & \multirow{2}{*}{$\begin{array}{c}\text { No. } \\
\text { ASOCIADOS }\end{array}$} & & & & \\
\hline & & Antes & Después & & Primaria & Básica Secundaria & Profesional & No Profesional \\
\hline \multirow{3}{*}{ Municipio 1} & Organización 1.1 & & $\mathrm{X}$ & 38 & $\mathrm{X}$ & & $\mathrm{X}$ & \\
\hline & \begin{tabular}{|ll} 
Organización 1.2 &
\end{tabular} & & $\mathrm{X}$ & 27 & $\mathrm{X}$ & & & $\mathrm{X}$ \\
\hline & Organización 1.3 & $\mathrm{X}$ & & 35 & $\mathrm{X}$ & & $\mathrm{X}$ & \\
\hline Municipio 2 & Organización 2.1 & & $\mathrm{X}$ & 20 & $\mathrm{X}$ & & $\mathrm{X}$ & \\
\hline Municipio 3 & Organización 3.1 & $\mathrm{X}$ & & 72 & $\mathrm{X}$ & & & $\mathrm{X}$ \\
\hline \multirow{3}{*}{ Municipio 4} & Organización 4.1 & $\mathrm{X}$ & & 20 & $\mathrm{X}$ & & & $\mathrm{X}$ \\
\hline & Organización 4.2 & & $\mathrm{X}$ & 72 & $\mathrm{X}$ & & $\mathrm{X}$ & \\
\hline & Organización 4.3 & & $\mathrm{X}$ & 25 & & $\mathrm{X}$ & $\mathrm{X}$ & \\
\hline Municipio 5 & Organización 5.1 & & $\mathrm{X}$ & 86 & $\mathrm{X}$ & & $\mathrm{X}$ & \\
\hline Municipio 6 & Organización 6.1 & & $\mathrm{X}$ & 43 & $\mathrm{X}$ & & $\mathrm{X}$ & \\
\hline Municipio 7 & Organización 7.1 & $\mathrm{X}$ & & 21 & $\mathrm{X}$ & & $\mathrm{X}$ & \\
\hline Municipio 8 & Organización 8.1 & & $\mathrm{X}$ & 20 & $\mathrm{X}$ & & $\mathrm{X}$ & \\
\hline \multirow{2}{*}{ Municipio 9} & \begin{tabular}{|l} 
Organización 9.1 \\
\end{tabular} & & $\mathrm{X}$ & 64 & $\mathrm{X}$ & & $\mathrm{X}$ & \\
\hline & Organización 9.2 & & $\mathrm{X}$ & 120 & $\mathrm{X}$ & & $\mathrm{X}$ & \\
\hline Municipio 10 & Organización 10.1 & & $\mathrm{X}$ & 108 & $\mathrm{X}$ & & $\mathrm{X}$ & \\
\hline Municipio 11 & Organización 11.1 & $\mathrm{X}$ & & 109 & $\mathrm{X}$ & & $\mathrm{X}$ & \\
\hline Municipio 12 & Organización 12.1 & & $\mathrm{X}$ & 17 & $\mathrm{X}$ & & $\mathrm{X}$ & \\
\hline Municipio 13 & Organización 13.1 & & $\mathrm{X}$ & 50 & $\mathrm{X}$ & & & $\mathrm{X}$ \\
\hline \multirow{2}{*}{ Municipio 14} & Organización 14.1 & & $\mathrm{X}$ & 110 & $\mathrm{X}$ & & $\mathrm{X}$ & \\
\hline & Organización 14.2 & $\mathrm{X}$ & & 35 & $\mathrm{X}$ & & $\mathrm{X}$ & \\
\hline Municipio 15 & Organización 15.1 & & $\mathrm{X}$ & 78 & $\mathrm{X}$ & & $\mathrm{X}$ & \\
\hline
\end{tabular}


considerados como requerimientos que los perjudican y que no les corresponde atender. Sobre este particular un asociado en un grupo focal dice:

La industria no viene a recoger la leche a los tanques de refrigeración, porque quieren llegar a donde haya al menos 2000 litros de leche. La gente ya no trae la leche al tanque porque prefiere venderle al crudero que le hace el favor de traerle la sal, el mercado y llevar el guarapo a los obreros.

El crudero al que hace referencia el asociado es el comprador informal, que en condiciones de bajos estándares sanitarios, recoge la leche por las fincas para comercializarla, también en un mercado informal de transformadores o consumidores finales. Los productores confieren significado a los favores recibidos por este actor del mercado, valoran el relacionamiento amistoso y la inexistencia de requerimientos de calidad.

En entrevista otro asociado refiriéndose al acopio de la leche expone: "a los grupos no nos sirvió porque empresas como Alquería sí ofrecen mejor precio, pero exigen altas calidades y los productores no alcanzamos esa calidad, así que preferimos producir en nuestras fincas y vender cada uno independiente".

Los esquemas interpretativos descritos aquí han sido construidos socialmente en la interacción y a través de la historia de estas comunidades, los mismos confluyen para que las organizaciones observadas se guarden alta homogeneidad en sus estructuras y formas de gestionarse; puede afirmarse que estas convergencias simbólicas sí se han convertido en los patrones de isomorfismo organizacional y no los arreglos institucionales de la competitividad en los que se soportan los programas de apoyo del gobierno referidos en esta investigación.

\section{Conclusiones}

Las estructuras de significación construidas socialmente por la comunidad de productores observada, no claudican necesariamente ante las presiones racionales de la eficiencia y productividad (Berger y Luckman, 1995; Meyer y Rowan, 1999, Zucker, 1999; Greenwood y Hinings, 1996)), por esta razón es necesario incorporar a los instrumentos de política pública un componente de innovación social (Westley y Antadze, 2010) para que haya un activo involucramiento de la comunidad y la justificación del cambio emerja de las comprensiones colectivas de las personas y no de prescripciones externas a las comunidades, de este modo es posible garantizar la sostenibilidad a mediano y largo plazo de los propósitos sociales (Kliksberg, 1998).

Las racionalidades que subyacen a las decisiones de las comunidades observadas son constructos sociales que compiten con las lógicas racionales de los instrumentos de política pública. Los cambios en el desempeño de los pequeños productores del eslabón primario de la industria láctea, esperado por los formuladores del conpes 3675 de 2010 no se logró, porque no ocurrió la construcción de sentido alrededor de los mitos racionales en los que descansan los dogmas del mercado. Los pequeños productores antepusieron sus temores y expectativas a los requerimientos de estándares de calidad, eficiencia en costos y competitividad. Se evidenció una convergencia en torno a la representación de que, si bien en principio hubo motivación porque el Estado llegaría con recursos, las condiciones competitivas de las organizaciones no cambiaron porque no se incorporó a las rutinas las nuevas prácticas propuestas.

Las tipificaciones en torno a su propia realidad, sus formas de vida, sus temores y expectativas, se mantienen en contradicción con los preceptos de las certificaciones y estándares de calidad, la eficiencia en costos y la maximización de la utilidad. En este panorama es indudable la mediación del factor educativo, dado que en la medida que haya capacidad de los individuos para interpretar su realidad en el contexto social, económico, técnico y político, se incrementan las capacidades para decidir autónomamente por el trabajo asociativo y compartir visiones de futuro (Suárez y Suárez, 2017).

Este conflicto entre lógicas se profundiza cuando se evidencian desacoplamientos (Weick, 2009) como la baja corresponsabilidad entre las expectativas y los ofrecimientos, o la ausencia de coordinación entre los niveles del gobierno.

En relación con lo teorizado por Dimaggio y Powell (1999), esta investigación aporta una evidencia empírica en el sentido de que los patrones institucionales del campo organizacional de la industria láctea en Boyacá (Colombia), no han alcanzado procesos de institucionalización en las organizaciones de pequeños productores del eslabón primario, en ellas se anteponen 
las tipificaciones sobre su realidad, construidas en la intersubjetividad. Ante la incertidumbre provocada por las alteraciones de los mercados como manifestación de la globalización, los sujetos interpretan su entorno de acuerdo con sus experiencias y equipamientos culturales, estas comprensiones son las generadoras de comportamientos que se repiten de manera crónica.

Las agendas de investigación que se vislumbran alrededor de estos fenómenos sociales son amplias, es preciso develar las fantasías que subyacen a las estructuras de significación en los grupos sociales y comprender el impacto de la educación, en un contexto sobreestructurado por los patrones institucionalizados del mercado.

\section{Referencias}

Alvarado, S. (2006). Metamorfosis de la concepción del cambio organizacional en el nuevo institucionalismo. Contaduría y Administración, 219, 9-40.

Berger, P. y Luckmann, T. (1995). La Construcción Social de la Realidad. Buenos Aires: Amorrortu editores

Bormann, E. (1983). Symbolic Convergence. Organizational Communication and culture. Comunication and organizations. An interpretative approach. Beverly Hills: Sage

Burrell, G. y Morgan, G. (1979). Sociological paradigms and organisational analisis. Londres: Heineman.

Consejo Nacional de Política Económica y Social, documento (Conpes) 3675 (2010). Departamento Nacional de Planeación. Recuperado de: https://www. minagricultura.gov.co/ministerio/direcciones/Documents/d.angie/conpes $\% 20$ 3675.pdf

Creswell, J. (2007). Qualitative Inquiry and Research Design: Choosing among five approaches. Thousand Oaks: Sage Publications.

Crozier, M. y Friedberg (1990). El actor y el sistema. Las contradicciones de la acción colectiva. México: Editorial Patria S.A. de C. V.

Cruz, A., Prado, C. y Díez, F. (2014). Por qué se institucionalizan las organizaciones. Revista Europea de Dirección y Economía de la Empresa, 23, 2230.

De la Rosa, A. (2002). Teoría de la Organización y Nuevo Institucionalismo en el Análisis Organizacional. Administración y Organizaciones, 4(8), 13-44.

Dimaggio, P. y Powell, W. (1999). Retorno a la Jaula de Hierro: el isomorfismo institucional y la racionalidad colectiva en los campos organizacionales. El Nuevo Institucionalismo en el Análisis Organizacional. México: Fondo de Cultura Económica.

Greenwood, R. y Hinings, C. (1996). Undestanding radical organizational change: bringing together the old and the new institutionalism. Academy of Management Review, 21(4), 1022-1054.

Ibarra, E. y Montaño, L. (1987). Mito y poder en las organizaciones. Un análisis crítico de la Teoría de la Organización. México: Trillas.

Jepperson, R. (1999). Refinamiento de la Teoría Institucional. Neoinstitucionalismo en el Análisis Organizacional.. México: Fondo de Cultura Económica
Kliksberg, B. (1998). Repensando el Estado para el desarrollo social: más allá de dogmas y convencionalismos. Revista Mexicana de Ciencias Políticas y Sociales, 43(173), 139-186.

March, J. (1994). Ambiguity and Interpretation. New York: The Free Press

Meyer, J. y Rowan, B. (1999). Organizaciones Institucionalizadas: La estructura formal como mito y ceremonia. El Nuevo Institucionalismo en el Análisis Organizacional. México: Fondo de Cultura Económica

Muñoz, R. (2010). Cambio y Cultura Organizacionales. Una crítica teórica y un estudio de caso. Sociología de la empresa. Del marco histórico a las dinámicas internas. Medellín: Siglo del Hombre Editores.

Selznick, P. (1949). TVA and the Grass Roots. Los Angeles: University of California Press

Simon, H. (1988). El comportamiento administrativo. Estudio de los procesos decisorios en la organización administrativa. Buenos Aires: Aguilar

Suárez, M. y Suárez, L. (2017). El conocimiento, determinante en procesos de asociatividad: una experiencia en conformación de redes empresariales. Encuentros 15(2), 83-97.

Tolbert, P. y Zucker, L. (1996). The institutionalization of institutional theory. Handbook of organization studies. London: Sage Publications.

Weber, M. (2002). Economía y Sociedad. México: Fondo de Cultura Económica.

Weick, K. (2009). Las organizaciones educativas como sistemas flojamente acoplados. (Traductor Caldera, D. y Ortega, M.). Gestión y Estrategia, 36, 93110.

Westley y Antadze (2010). Making a Difference: Strategies for Scaling Social Innovation for Greater Impact. The Innovation Journal: The Public Sector Innovation Journal, 15(2), 1-19.

Zucker, L. (1999). El papel de la institucionalización en la persistencia cultural. El nuevo institucionalismo en el análisis organizacional. México: Fondo de Cultura Económica. 